\title{
L'ORGANISATION ET LE FONCTIONNEMENT DE LA PROFESSION D'HUISSIER DE JUSTICE EN REPUBLIQUE DEMOCRATIQUE DU CONGO : ETAT DES LIEUX ET ENJEUX ACTUELS
}

\author{
Par ABDOU MUHIMA Moïse*
}

\begin{abstract}
RESUME
Cet article s'interroge sur l'opportunité de la libéralisation de la profession d'huissier de justice en RDC et du rôle que joue l'huissier de justice dans l'administration de la justice pour la promotion ou la consolidation de l'Etat de droit en RDC. Avant la loi organique de 2016 sur la profession d'huissier de justice, cette dernière ne bénéficiait pas des garanties nécessaires pour jouer son rôle de profession juridique de proximité et sociale. Avec sa libéralisation, cette profession est structurée au tour de la Chambre nationale et de la chambre provinciale des huissiers de justice. Chacune de ses structures est composée d'une Assemblée générale, d'un Bureau et d'un Commissariat aux comptes. Ces différents organes veillent à l'efficacité et l'intégrité de la profession. Cette reforme permet aux huissiers de justice de s'organisent au tour d'une corporation autonome et indépendante bénéficiant de garantie nécessaire pour contribuer efficacement à la bonne administration de la justice et la consolidation de l'Etat de droit en RDC.
\end{abstract}

\begin{abstract}
This article discusses the opportunity of the liberalization of the occupation of enforcement agent (Bailliff) in DRC and the role played by the bailliff in the administration of justice for the promotion and the consolidation of the Rule of law in DRC. Before the organic law of 2016 on the occupation of enforcement agent, they did not profit from the necessary guarantees to play its role of legal profession of proximity and social. With its liberalization, this profession is structured in the national and provincial chambers of enforcement agent. Each of its structures is composed of a General Assembly, an Office and an Auditor. These various bodies ensure the efficiency and integrity of the profession. And, This reform allows bailiffs to organize themselves into an autonomous and independent corporation benefiting
\end{abstract}

* Moïse Abdou Muhima est actuellement candidat au Diplôme d'études supérieures (DES) en Droit public à la Faculté de droit de l'Université de Kinshasa (RDC). Avocat au Barreau de Kinshasa / Matete. Chercheur au Centre de Recherches et d'Etudes sur l'Etat de Droit en Afrique (CREEDA) et Membre du CODESRIA. Ses recherches portent spécialement sur l'intégration africaine, le règlement des différends internationaux et l'Etat de droit en Afrique. E-mail : muhimamoise@gmail.com et moisem@creeda-rdc.org. 
from the necessary guarantee to contribute effectively to the good administration of justice and the consolidation of the Rule of law in the DRC.

\section{INTRODUCTION}

L'huissier de justice est un fonctionnaire chargé de l'exécution des tâches qui s'adressent aux personnes étrangères à la juridiction. C'est à lui qu'appartient principalement le devoir d'instrumenter les exploits et d'exécuter les décisions de justice. Comme l'indique le mot, un huissier est un fonctionnaire chargé des ports. Du latin « huis », qui signifie « porte », ce mot servait initialement à désigner un préposé d'un tribunal qui devait ouvrir les portes à l'entrée de la composition. A nos jours, il est actif au sein d'un ordre ou d'un corps soumis à des règles strictes censées le déterminer à observer certaines règles indispensables à ses fonctions en tout temps, envers tous et en tout lieu ${ }^{1}$.

En RDC, les huissiers de justices sont des auxiliaires de la justice qui concourent dans l'administration de celle-ci. Ils concourent à la bonne administration de la justice en apportant une aide essentielle, voire indispensable, aux magistrats. Leur contribution au respect des délais dans les procédures judiciaires est indispensable pour le bon fonctionnement de l'appareil judiciaire. Ainsi, les huissiers de justice participent dans l'élaboration et la signification des exploits et des actes ${ }^{2}$.

Avant 2016, cet office n'était pas libéralement organisé par la loi. Jadis, les chefs des juridictions prenaient le soin de désigner des individus parmi le personnel administratif de la juridiction pour accomplir les fonctions d'huissier de justice. Il eu fallu attendre la loi $\mathrm{n}^{\circ}$ 16/011 du 15 juillet 2016 portant création et organisation de la profession d'huissier de justice pour que cette profession soit libéralisée en $\mathrm{RDC}^{3}$. D'une manière générale, les professions libérales peuvent être définies comme des prestataires de services à caractère intellectuel, indépendants et responsables qui exercent en l'absence de lien de subordination et qui ont l'obligation de respecter le secret professionnel.

De façon plus pragmatique, deux critères objectifs permettent de considérer qu'une profession est libérale : d'une part, le critère d'ordre social (exercice d'une activité non salariée) et, d'autre part, le critère d'ordre statutaire c'est-à-dire qu'en ce qui concerne les huissiers de justices, ils ne sont pas soumis au statut de la fonction publique mais plutôt soumis aux textes juridiques qui organisent leur corporation.

1 Martiza FELICES-LUNA, La Justice en République Démocratique du Congo : transformation ou continuité ?, Champ pénal, Vol. VII, 2010 disponible sur https://doi.org/10.4000/champpenal.7827 consulté le 04 janvier 2020.

2 Emmanuel J. LUZOLO BAMBI LESSA, Traité de droit judiciaire. La justice congolaise et ses institutions,

Kinshasa, 2018.

3 Loi n 16/011 du 15 juillet 2016 Portant création et organisation de la profession d'huissier de justice, JORDC, 57è année, Numéro spécial du 30 juillet 2016. 
Au demeurant, cet article s'intéresse donc à l'organisation actuelle de cette profession, les défis auxquels il est confronté et les perspectives d'avenir pour que le ministère d'huissier de justice puisse contribuer à la promotion et à la consolidation de l'Etat de droit en RDC

\section{A. L'Organisation de la profession d'huissier de justice}

En RDC de la profession d'huissier de justice est régi par la loi n¹6/011 du 15 juillet 2016 portant création et organisation de la profession d'huissier de justice. Cette loi a notamment le mérite d'avoir libéralisé cette profession en battant en branche les prescrits de la loi organique $\mathrm{n}^{\circ} 13 / 011$-B du 11 avril 2013 portant organisation, fonctionnement et compétences des juridictions de l'ordre judiciaire. Cette dernière plaçait les huissiers de justice sous le régime du personnel de carriers des services public de l'Etat en ces termes :

Sont agents de l'ordre judiciaire : les fonctionnaires et agents administratifs des greffes, des secrétariats des parquets, des services de la police .judiciaire des Parquets ainsi que les huissiers, lorsque ceux-ci sont de carrière. Ils sont tous régis par le statut du personnel de carrière des services publics de l'État ${ }^{4}$.

Ainsi, sous ce régime, les huissiers de justice été placés sous une double autorité et ne semblaient devoir se réclamer d'aucune autonomie. Ils étaient sous l'autorité administrative du chef de la juridiction à laquelle ils sont affectés après avoir été nommé par arrêté ministériel comme agent de l'ordre judiciaire ${ }^{5}$.

A la différence de l'ancien régime où l'huissier de justice était soumis au statut de fonctionnaire de l'Etat, à partir du 15 juillet 2016 il exerce une profession libérale, autant que les avocats. La loi nº16/011 du 15 juillet 2016 prévoit la coexistence d'une chambre nationale et des chambres provinciaux des huissiers de justice en RDC $^{6}$.

\section{La chambre nationale des huissiers de justice}

La chambre nationale des huissiers de justice a son siège à Kinshasa, son ressort est l'ensemble du territoire national ${ }^{7}$ et représente l'ensemble de la profession auprès des pouvoirs

4 Article 3 de la loi organique ${ }^{\circ} 13 / 011-\mathrm{B}$ du 11 avril 2013 portant organisation, fonctionnement et compétences des juridictions de l'ordre judiciaire.

5 Emmanuel J. LUZOLO BAMBI LESSA, La détention préventive en procédure pénale zaïroise, tome II, Contribution à l'efficacité de la détention préventive, Thèse de doctorat en droit, Université de droit, d'économie et des sciences d'Aix-Marseille, Faculté de droit et de science politique d'AixMarseille, 1996, p.338.

6 Article 18 de la loi nº $16 / 011$ du 15 juillet 2016 portant création et organisation de la profession d'huissier de justice, note 4 .

7 Article 18 alinéa $1^{\text {er }}$ de la loi nº $16 / 011$ du 15 juillet 2016 , note 4 . 
publics et des tiers ${ }^{8}$. Et, elle est dotée de la personnalité juridique. Ses organes sont l'Assemblée générale, le Bureau et le Commissariat aux Comptes ${ }^{9}$. La Chambre nationale des huissiers de justice veuille à la gestion, à la discipline générale et à l'honneur de la profession d'huissiers de justice. Ses organes sont l'Assemblée générale, le Bureau et le Commissariat aux comptes.

Elle est compétente pour établir un code de déontologie à soumettre à l'approbation du ministère ayant la justice dans ses attributions ; fixer le tarif minimum et maximum des honoraires et des frais de la profession à soumettre à l'approbation du ministère ayant la justice dans ses attributions ; préparer le budget de la profession et le faire adopter par l'Assemblée générale ; vérifier la tenue de la comptabilité dans les études des huissiers de justice ; veuillez à la formation permanente des huissiers de justice et des clercs, en concertation avec le ministère ayant la justice dans ses attributions ; donner des avis lorsqu'elle en est requise sur les questions intéressant la profession ; délivrer les attestations de moralité et les cartes professionnelles ; prévenir, concilier et, le cas échéant, statuer au premier degré ou au degré d'appel, selon le cas, sur tous les différends d'ordre professionnel pouvant survenir dans la profession des huissiers de justice ; connaitre au deuxième degré les décisions rendues par les chambres provinciales; et élaborer le règlement intérieur à soumettre à l'approbation du ministère ayant la justice dans ses attributions et donner son avis sur le règlement intérieur des chambres provinciales.

\section{La chambre provinciale des huissiers de justice}

La Chambre provinciale représente l'ensemble des huissiers de justice du ressort de la Cour d'appel auprès du pouvoir public et des tiers. Cette chambre est composée d'une assemblée générale, du Bureau et du Commissariat aux comptes ${ }^{10}$. L'Assemblée générale de la Chambre provinciale des huissiers de justice est composée de tous les huissiers de justice $\mathrm{du}$ ressort ${ }^{11}$. Elle fonctionne en différentes Commissions chargée des questions spécifiques qui touchent à la profession d'huissier de justice. Ces différentes commissions sont créées et fonctionnent conformément au Règlement intérieur de la Chambre provinciale des huissiers de justice ${ }^{12}$.

Le Bureau est l'organe dirigeant de la Chambre Provinciale. Il assure l'exécution des décisions de l'Assemblée Générale. Il est l'organe d'administration de la Chambre. Cet organe veuille à l'exécuter les décisions de l'Assemblée générale, assurer la gestion quotidienne de la chambre et élabore le bilan de l'exercice annuelle. Il établir tous les documents

8 Article 19 alinéa $1^{\text {er }}$ de la loi $n^{\circ} 16 / 011$ du 15 juillet 2016 , note 4 .

9 Article 18 de la loi n $16 / 011$ du 15 juillet 2016, in fine, note 4.

10 Article 18 de la loi ${ }^{\circ} 16 / 011$ du 15 juillet 2016 , note 4.

11 Article 22 de la loi n¹6/011 du 15 juillet 2016, note 4.

12 Pour la Chambre provinciale des huissiers de justice de Kinshasa/Gombe notamment, son règlement intérieur prévoit la Commission disciplinaire ( Articles 56-58), La Commission de Recherche (Article 59-61) et la Commission électorale (Article 62-63). 
devant être soumis à l'Assemblée Général et proposer les amendements du Règlement intérieur à l'Assemblée Générale. Il assurer, dans son ressort, l'exécution des décisions prises par la Chambre Nationale et veiller à la stricte observation des devoirs des huissiers de justice ainsi qu'à la protection de leur droit. Le Bureau examine toutes réclamations de la part des tiers contre les huissiers à l'occasion de l'exercice de leur fonction, et notamment en ce qui concerne la taxe des frais et délivrer ou refuse de délivrer, par une décision motivée, les certificats de moralité demandés par les stagiaires.

Le commissariat au compte est composé du commissaire au compte, du commissaire au compte adjoint et du rapporteur. Les membres du commissariat au compte sont élus par l'assemblée générale. Le commissariat au compte a pour mission principale de certifier les comptes trimestriels du bureau et des études en réalisant un audit. Ainsi donc, pour mener à bon port sa mission, le bureau et les études ont l'obligation de mettre à sa disposition tout document nécessaire à sa mission. L'audit est requis à tout moment si besoin se présente et à l'issue de chaque audit, le commissariat au compte dresse des procès-verbaux et un rapport général en est fait lors de l'assemblée générale.

\section{B. Défis dans la pratique et enjeux actuels}

Le fonctionnement de l'office de l'huissier de justice est actuellement confronter à plusieurs défis. Ces derniers découlent de la nature même de cette profession ${ }^{13}$. Etat une profession libérale, elle dépend de la bonne gestion du corps des huissiers de justice. Il reste à s'interroger sur les garanties du bon fonctionnement de ce ministère et les enjeux majeur pour que ce ministère contribue à la consolidation de l'Etat de droit en RDC.

\section{Garanties du bon fonctionnement de la profession d'huissier de justice}

Le bon fonctionnement d'une profession dépend de plusieurs paramètres. Ces paramètres peuvent être objectifs ou subjectifs. Ainsi ils tiennent des qualités ou de l'état des individus qui constituent le corps de la profession mais aussi des paramètres organisations et de l'environnement dans la quelle est exercé cette profession. La libéralisation de la profession d'huissier de justice telle qu'elle est organisation par la loi n¹6/011 du 15 juillet 2016 portant création et organisation de la profession d'huissier de justice en République démocratique du Congo a consacré l'autonomie et la garantie d'une indépendance au profit des huissiers de justice.

13 Article $1^{\text {er }}$ de la loi ${ }^{\circ} 16 / 011$ du 15 juillet 2016 , note 4 . 


\section{Autonomie de la profession et de ses membres}

La nature et l'organisation actuelles de la profession d'huissier de justice offre plus des garanties pour le bon fonctionnement de cet office qu'avant l'entrée en vigueur de la loi organique de 2016.

En effet, avant la loi organique de 2016, la dépendance hiérarchique de l'huissier de justice était perceptible dans la pratique. L'huissier de justice était soumis au statut du personnel de carrière des services publics de l'État, il était fonctionnaire de l'Etat. Le Chef de la juridiction qui s'occupé de la mise en service des huissiers de justice dans son ressort était son l'autorité hiérarchique immédiate puisque celui-ci est responsable de la structure dont dépend immédiatement l'agent ${ }^{14}$. La pratique voulait à ce que ceux-ci soient sous l'autorité hiérarchique du chef de juridiction à la quelle ils sont rattachés. Dans ce contexte, l'indépendance et l'autonomie de l'huissier de justice trouvait des limites à plusieurs égards : du pourvoir du chef de la juridiction et du pouvoir exécutif ${ }^{15}$.

En sa qualité de fonctionnaire de l'Etat, l'huissier de justice ne devrait pas dépendre financièrement des honoraire et débours perçus auprès des justiciables pour les différentes prestations car les fonctionnaires de l'Etat ont une contrepartie pécuniaire du travail fourni que reçoit mensuellement tout fonctionnaire de l'Etat à terme échu et qui comprend le salaire de base et, le cas échéant, les primes et indemnités qui relève du budget de l'Etat ${ }^{16}$. Dans ce contexte, sur le plan financier, l'huissier de justice ne pouvait pas non plus se prévaloir d'une certaine autonomie financière directe à l'égard du pouvoir exécutif. Mais actuellement, avec la libéralisation de cette profession ${ }^{17}$, l'huissier de justice dans l'exercice de sa profession, il a droit aux honoraires et débours qui sont fixés de commun accord entre lui et son client dans le cadre d'un tarif minimum et maximum déterminé par la chambre nationale des Huissiers de justice ${ }^{18}$. Cette nouvelle situation est un atout important pour l'autonomisation financière de l'huissier de justice qui a une incidence positive sur son indépendance et son efficacité dans l'exercice de sa profession ${ }^{19}$.

\section{Pas de dépendance hiérarchique}

La profession d'huissier de justice est constituée d'un corps organisé et structuré d'huissiers de justice. Ce corps est structuré à deux niveaux : au niveau national il y a une chambre

14 Article 3 de la loi n ${ }^{\circ}$ 16/013 DU 15 Juillet 2016 Portant statut des agents de carrière des services publics de l'Etat, JORDC, $57^{\mathrm{e}}$ année, Numéro spécial du 3 aout 2016.

15 BOSLY H.D. et David VANDERMEERSCH, Droit de la procédure pénale, La Charte, 2005.

16 Pierre Akele Adau et Angélique Sita Muila Akele, «Quelle stratégie pour combattre la corruption en RDC ? », in Pierre Akele Adau (dir.), Réforme du Code pénal congolais. A la recherche des options fondamentales du Code pénal congolais, Kinshasa, Editions du Cepas, 2008.

17 Article $1^{\text {er }}$ de la loi n ${ }^{\circ} 16 / 011$ du 15 juillet 2016 , note 4 .

18 Article 19 alinéa 2 (2) de la loi n ${ }^{\circ} 16 / 011$ du 15 juillet 2016, note 4 .

19 Michel De Villiers et Armel Le Divellec, Dictionnaire du droit constitutionnel, Paris, 9ème édition, Paris Sirey, 2013. 
nationale qui représente l'ensemble de la profession auprès des pouvoirs publics et des tiers et au niveau de chaque province constituant la compétence territoriale de la Cour d'appel il y a des chambres provinciales. L'Assemblé général, le Bureau et le Commissariat aux comptes sont des organes de la Chambre nationale des huissiers de justice, et qu'on retrouve aussi dans chaque structure provinciale des huissiers de justice ${ }^{20}$.

Il n'existe pas un lien de subordination qui implique l'exécution d'un travail sous les ordres et pour le compte d'un employeur entre les animateurs des différents organes et les autres huissiers de justice. Ces derniers exercent en toute indépendance leurs fonctions dans le seul respect des lois de la République. L'huissier de justice dirige son étude seul ou en association. Lorsqu'il est seul, l'Huissier de justice, titulaire de l'étude coordonne toutes les activités de l'étude. Dans l'exercice de son ministère, aucune procédure ne peut être entamée sans qu'il en prenne connaissance et donne son avis favorable. En cas d'association, la gestion du cabinet est réglée par un contrat d'association ${ }^{21}$. Seul cet engagement détermine les modalités pratiques de son ministère et lui permet de n'être soumis à aucune autorité dans l'exercice de sa profession.

Les animateurs des organes de la Chambre nationale ou provinciale ne sont pas des supérieurs hiérarchiques des huissiers de justice du ressort. Le Président de la Chambre nationale n'est que le premier d'entre les huissiers de justice sur toute l'étendue du territoire national. De même, le Président de la chambre provinciale en ce qui concerne les huissiers de justice de son ressort. L'un ou l'autre n'a aucune autorité à admonester autres huissiers de justice. Il n'y a pas de lien de subordination entre les différents organes qui composent la chambre provinciale des huissiers de justice. Moins encore, la chambre nationale n'a aucun pouvoir d'injonction sur les organes d'une chambre provinciale ni sur un Huissier de justice dans l'exercice de ses fonctions.

Tout de même, il n'en demeure pas moins vrai qu'il existe des mécanismes de collaboration entre les différents organes de chaque structure. Il peut y être distingué d'une part, une collaboration verticale qui concerne les relations entre les chambres provinciales et la chambre nationale. Et d'autre part, une collaboration horizontale entre les différents organes de chambres (Nationale ou provinciales).

\section{La collaboration structurelle et organique}

Dans l'exercice de leurs fonctions, les chambres provinciales collaborent avec la chambre nationale. Pour sa constitution, l'Assemblée générale de la Chambre nationale est composée des membres des bureaux des chambres provinciales. Ceci est le point de départ de la collation et de la prise en considération des opinions des chambres provinciales par la chambre nationale. Cette dernière établi un code de déontologie dont, après avoir été soumis à l'ap-

20 Article 18 de la loi nº $16 / 011$ du 15 juillet 2016 , note 4 .

21 Article 9 du Règlement intérieur de la Chambre provinciale des huissiers de justice de Kinshasa / Gombe. 
probation du ministère ayant la justice dans ses attributions, le respect est suivi par les chambres provinciales qui font rapport régulièrement à la Chambre nationale à ce sujet. De même, après que la Chambre nationale ai fixé le tarif minimum et maximum des honoraires et des frais de la profession, les organes des chambres provinciales, doivent veiller au respect de ce tarif par tous les huissiers de justice de leurs ressorts respectifs et les différents commissariats aux comptes provinciaux vérifient les comptabilités des études de leurs ressorts et en fait rapport à la chambre nationale.

Il sied en outre de constater qu'en dépit du fait que les huissiers de justices et les clercs pour leur entrée en fonction prêtent serment dans la Cour d'appel de leurs ressort respectifs, c'est la chambre nationale qui met à la disposition des chambres provinciales les cartes professionnelles à délivrer aux huissiers de justice de leurs ressorts. Mais également la chambre nationale sert de juridiction d'appel pour les décisions rendues par les chambres provinciales. Les chambres provinciales dans leurs ressorts respectifs assurent l'exécution des décisions prises par la Chambre Nationale.

Dans une chambre provinciale des huissiers de justice, le Bureau de la Chambre est l'organe administratif chargé d'exécuter les décisions de l'Assemblée générale constituée par tous les huissiers de justice du ressort ${ }^{22}$. Pour assurer la formation continue des huissiers, les membres du bureau de la chambre provinciale collaborent étroitement avec tous les huissiers de justice de la juridiction pour s'assurer de leur assistance et de leur participation active à la fonction. Le bureau veuille à prévenir ou concilier tous les différents d'ordre professionnel entre les huissiers.

\section{Enjeux actuel : une mission indispensable pour la consolidation de l'Etat de droit}

L'huissier de justice est un officier ministériel ayant le monopole de certaines activités. Il est à la fois juriste de proximité et chef d'entreprise. Il est donc doublement compétent pour conseiller l'entreprise et le citoyen ${ }^{23}$. L'Etude de l'huissier de justice comprend des assistants juridiques spécialisés, des comptables et des secrétaires, ainsi que tout autre spécialiste engagé pour le fonctionnement de cette grande entreprise qui s'occupe des questions juridiques.

La majeur partie du travail de l'huissier de justice est repartie entre la rédaction des actes et l'assistance aux justiciables. Il informe les justiciables qu'une action en justice est engagée contre eux en leur remettant une convocation dite assignation pour une affaire civile (ex: divorce) ou dite citation dans le cas d'une affaire pénale. Une fois le jugement rendu, l'huissier informe les justiciables des décisions de justice ${ }^{24}$. Ainsi l'huissier de justice doit faire à un double challenge : celui d'informer et celui de conseiller les justiciables.

22 Article 22 de la loi n $16 / 011$ du 15 juillet 2016, note 4.

23 Likulia Bolongo, Droit et science pénitentiaires : Vers un traitement scientifique de la délinquance au Zaïre, Paris, 1981.

24 Article 13 de la loi nº16/011 du 15 juillet 2016, note 4 . 
Le principal devoir qui pèse sur l'huissier de justice est le devoir de conseil les justiciables. Il se doit d'informer et d'éclairer ses clients. En effet, il est de plus en plus difficile pour les citoyens de se tenir au fait de la loi, et d'en comprendre toutes la portée. L'huissier de justice se doit alors, de donner l'information la plus complète à son client. Il est tenu d'assurer l'efficacité des actes pour lesquels il est requis et de conseiller son client, qu'il soit demandeur ou défendeur.

Mais aussi, la transmission des informations est une activité capitale de l'huissier de justice dans le cadre de son rôle judiciaire et social. Il doit non seulement informer les parties des actes de procédure qui les concernent, mais aussi et surtout informer des conséquences qui en découlent. Il est tenu d'un devoir général de conseil et d'information envers les destinataires de ses actes : il doit fournir une information complète, loyale et précise et doit conserver une neutralité absolue vis-à-vis des justiciables. L'huissier a un rôle d'écoute afin d'éviter les procédures trop coûteuses et d'information des débiteurs sur leurs droits.

Il veille à l'exécution des décisions judiciaires en ce que la présence de l'huissier de justice est fondamentale pour que les jugements rendus par la justice soient appliqués, notamment en ce qui concerne les recouvrements de créances (ex : loyers, pensions alimentaires, factures...). Dans la majorité des cas, cette exécution se fait à l'amiable, sans heurt. L'huissier a pour objectif principal de conseiller et de rechercher des solutions avec la personne jugée. Il proposera par exemple des plans de remboursement des créances. C'est en dernier recours et dans le cas d'une absence d'accord que l'huissier après décision de justice procèdera à une expulsion (impayé de loyers) ou à une saisie dans le cadre d'un recouvrement forcé des dettes (impayés de factures, de pensions alimentaires...).

Autre fonction essentielle de l'huissier est d'établir des constats qui ont valeur de preuve incontestables devant les tribunaux et permettent aux victimes d'un préjudice d'obtenir réparation. Il peut s'agir notamment des dégâts des eaux, du défaut de construction ou d'accidents de travail. L'huissier de justice peut aussi être amené à effectuer des consultations juridiques pour organiser une vente aux enchères, valider le règlement d'un jeu ou tout autre activité lui confiée par la loi ${ }^{25}$.

Il demeure sans nul doute qu'au vue de sa technicité, les citoyens ne peuvent pas se passer des services de l'huissier de justice puisque celui-ci se trouve au cœur des procédures judicaires. Ainsi, les questions de droit étant au cœur de l'Etat de droit, l'accompagnement de l'huissier de justice aux côté des citoyens contribue énormément à l'accès à la justice par ces derniers et participe à la consolidation de l'Etat de droit.

\section{CONCLUSION}

Apparu au moyen-âge et visiblement peu méconnue de nos jours, la profession d'huissier de justice est souvent associée dans l'imaginaire collectif au coup de sonnette tant redouté,

25 Serge Guinchard, Gabriel Montagnier, André Varinard et Thierry Debard, Institutions juridictionnelles, Paris, 12ème édition, Dalloz, 2013. 
achevant de placer les personnes en difficulté dans une situation de plus grand dénuement ${ }^{26}$. Cet article a démontré que ce serait pourtant une erreur de réduire la profession d'huissier de justice à cette seule dimension coercitive qui est loin d'être l'activité la plus importante de ce juriste pourtant de proximité. Les huissiers de justice sont très proches de la population à telle enseigne que la mission de ces professionnels de droit s'avère sociale.

En RDC, 'administration de la justice et ses faiblesse actuelle ont un impact négatif sur le fonctionnement de l'appareil judiciaire et comptent parmi les maux qui gangrènent ce dernier. Dans cet article il a été mentionné que les huissiers de justice ont été jusqu'à un certain moment récent, désigné par les présidents des juridictions parmi les fonctionnaires et agents de carrière des services publics de l'Etat mis à leur disposition. Cette époque a été caractérisée par l'insuffisance de formation mais aussi l'absence de contre sur ses activités et de l'impunité qui s'en suivait. Ainsi, les formes de cette profession par la loi de 2016 ont consisté principalement à libéraliser le ministère de l'huissier de justice le mentant ainsi face à ces responsabilité en ce qu'il réponde de ses actes devant sa corporation et devant les instances judicaires.

\section{BIBLIOGRAPHIE}

Constitution de la République Démocratique du Congo, JORDC, $47^{\text {ème }}$ année, Numéro spécial du 18 février 2006.

Loi $\mathrm{n}^{\circ}$ 16/011 du 15 juillet 2016 Portant création et organisation de la profession d'huissier de justice, JORDC, 57è année, Numéro spécial du 30 juillet 2016.

Loi organique n $13 / 011-\mathrm{B}$ du 11 avril 2013 portant organisation, fonctionnement et compétences des juridictions de l'ordre judiciaire.

Loi $\mathrm{n}^{\circ}$ 16/013 du 15 Juillet 2016 Portant statut des agents de carrière des services publics de l'Etat, JORDC, $57^{\mathrm{e}}$ année, Numéro spécial du 3 aout 2016.

Ordonnance-loi n82-020 du 31 mars 1982 portant Code de l'organisation et de la compétence judiciaires, Journal officiel de la République démocratique du Congo, $47^{\mathrm{ème}}$ année, numéro spécial, 20 juin 2006.

Martiza FELICES-LUNA, La Justice en République Démocratique du Congo : transformation ou continuité ?, Champ pénal, Vol. VII, 2010 disponible sur https://doi.org/10.4000/champpenal.7827 consulté le 04 janvier 2020.

Emmanuel J. LUZOLO BAMBI LESSA, Traité de droit judiciaire. La justice congolaise et ses institutions, Kinshasa, 2018.

Béatrice Thomas-Tual, Droit de la fonction publique de l'Etat, Paris, Ellipses, 2005.

Bernard Durand, «Querelles doctrinales et « frontières », Cahiers des écoles doctorales, Les controverses doctrinales, Cahier des Ecoles doctrinales, $n^{\circ} 1$, mars 2000, Faculté de droit de l'Université Montpellier I, pp.9-16.

Chambliss, W.J., 1969, Crime and the Legal Process, New York, McGraw-Hill.

26 Denis Alland et Stéphane Rials (dir.), Dictionnaire de la culture juridique, Paris, 2014. 
Emmanuel J. LUZOLO BAMBI LESSA, La détention préventive en procédure pénale zaïroise, tome II, Contribution à l'efficacité de la détention préventive, Thèse de doctorat en droit, Université de droit, d'économie et des sciences d'Aix-Marseille, Faculté de droit et de science politique d'AixMarseille, 1996, p.338.

BOSLY H.D. et David VANDERMEERSCH, Droit de la procédure pénale, La Charte, 2005.

Pierre Akele Adau et Angélique Sita Muila Akele, «Quelle stratégie pour combattre la corruption en RDC ? », in Pierre Akele Adau (dir.), Réforme du Code pénal congolais. A la recherche des options fondamentales du Code pénal congolais, Kinshasa, Editions du Cepas, 2008.

Michel De Villiers et Armel Le Divellec, Dictionnaire du droit constitutionnel, Paris, 9ème édition, Paris Sirey, 2013

Règlement intérieur de la Chambre provinciale des huissiers de justice de Kinshasa / Gombe.

Likulia Bolongo, Droit et science pénitentiaires : Vers un traitement scientifique de la délinquance au Zaïre, Paris, 1981.

Serge Guinchard, Gabriel Montagnier, André Varinard et Thierry Debard, Institutions juridictionnelles, Paris, 12ème édition, Dalloz, 2013.

Denis Alland et Stéphane Rials (dir.), Dictionnaire de la culture juridique, Paris, 2014. 